\title{
Super-resolved time-frequency analysis of wideband backscattered data
}

\author{
Moore, John; Ling, $\mathrm{H}$.
}

Published in:

I E E E Transactions on Antennas and Propagation

Link to article, DOI:

$10.1109 / 8.387179$

Publication date:

1995

Document Version

Publisher's PDF, also known as Version of record

Link back to DTU Orbit

Citation (APA):

Moore, J., \& Ling, H. (1995). Super-resolved time-frequency analysis of wideband backscattered data. I E E E Transactions on Antennas and Propagation, 43(6), 623-626. https://doi.org/10.1109/8.387179

\section{General rights}

Copyright and moral rights for the publications made accessible in the public portal are retained by the authors and/or other copyright owners and it is a condition of accessing publications that users recognise and abide by the legal requirements associated with these rights.

- Users may download and print one copy of any publication from the public portal for the purpose of private study or research.

- You may not further distribute the material or use it for any profit-making activity or commercial gain

- You may freely distribute the URL identifying the publication in the public portal

If you believe that this document breaches copyright please contact us providing details, and we will remove access to the work immediately and investigate your claim 


\section{Communications}

\section{Super-Resolved Time-Frequency Analysis of Wideband Backscattered Data}

John Moore and Hao Ling

\begin{abstract}
A time-frequency super-resolution procedure is presented for processing wideband backscattered data containing both scattering center and natural resonance information. In this procedure, Prony's method is first applied in the frequency domain to locate scattering centers. The data is processed one slice at a time through the use of a sliding window function. Parameterized models for the scattering centers are obtained by a weighted average of the results from each segment. A similar procedure is used in the time domain to fully parameterize the natural resonances. In contrast to other time-frequency techniques, the time-frequency display from the present super-resolution procedure is not constrained in resolution by the Fourier limit.
\end{abstract}

\section{INTRODUCTION}

Time-frequency techniques such as the short-time Fourier transform and wavelet analysis have recently been used to process wideband radar echo from complex targets [1]-[4]. These techniques process the data one small chunk at a time through the use of a sliding window function. In the two-dimensional time-frequency plane, scattering centers, natural resonances, and dispersive phenomena can be simultaneously displayed to provide additional insights into the scattering mechanisms not available in either the time or the frequency domain. The additional insights gained in the timefrequency plane come, however, at the price of loss of resolution. For instance, when the short-time Fourier transform is applied to the data, the time resolution in the time-frequency display is limited by the frequency-extent of the sliding window function, not by the available frequency bandwidth of the signal. The processing of the data within each time or frequency window using super-resolution techniques such as Prony's method [5] or the ESPRIT algorithm [6] therefore appears quite attractive. It retains the advantage of simultaneous timefrequency display while overcoming the Fourier-limited resolution issue. In this paper, we describe a super-resolution procedure based on Prony's method for achieving parameter estimation of both scattering centers and natural resonances in the time-frequency plane.

Prony's method has been extensively applied to electromagnetic parameter estimation problems involving either time or frequency domain data. It has been used to extract the natural resonances contained in transient data in [5] and [7]. It has also seen success in the extraction of scattering centers from frequency data [8]; however, Prony's processing tends to perform rather poorly when both resonances and scattering centers are present at the same time, as is the case when wideband scattering data from complex targets are encountered

In our time-frequency super-resolution procedure, Prony's extraction is applied first in the frequency domain to locate scattering

Manuscript received February 22, 1994; revised February 24, 1995. This work is supported by the Joint Services Electronics Program Contract AFOSR F49620-92-C-0027.

J. Moore is with the Electromagnetics Institute, Technical University of Denmark, DK-2800 Lyngby, Denmark.

$\mathrm{H}$. Ling is with the Department of Electrical and Computer Engineering, The University of Texas at Austin. Austin, TX 78712-1084 USA

IEEE Log Number 9411455.

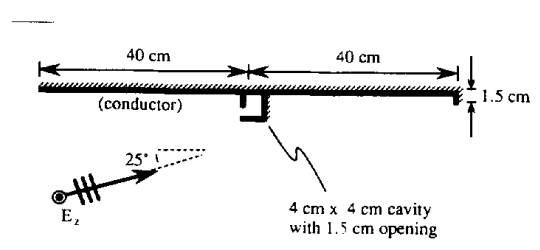

Fig. 1. Geometry of the conducting strip with cavity and fin.

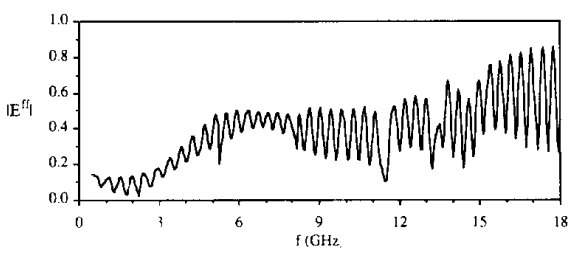

(a)

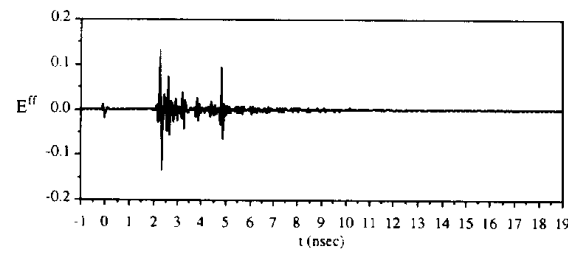

(b)

Fig. 2. Resulting backscattering from the strip of Fig. 1: (a) frequency domain. (b) time domain.

centers. We break up the wideband data into many small overlapping segments of narrow band data and repeatedly apply Prony's method. The results for each segment are weighted according to error and then averaged together. This prevents resonances, which will cause Prony's method to fail for a small number of segments, from corrupting the overall scattering center estimates. Once the scattering centers are found, a similar procedure is used in the time domain to locate the resonances. The backscattering from a conducting strip containing a resonant cavity will be used to demonstrate our approach.

\section{Simulation}

Fig. 1 shows a perfectly conducting strip containing a resonant cavity near the middle of the strip. A small fin exists at the right edge of the strip. Although this target is simple, its scattering is representative of signatures from more realistic targets with both exterior skinline contributions and subskinline resonances. For $E$-polarized incidence at 25 degrees from edge-on, three scattering centers due to the left edge, the cavity exterior, and the fin at the right edge will

0018-926X/95\$04.00@1995 IEEE 

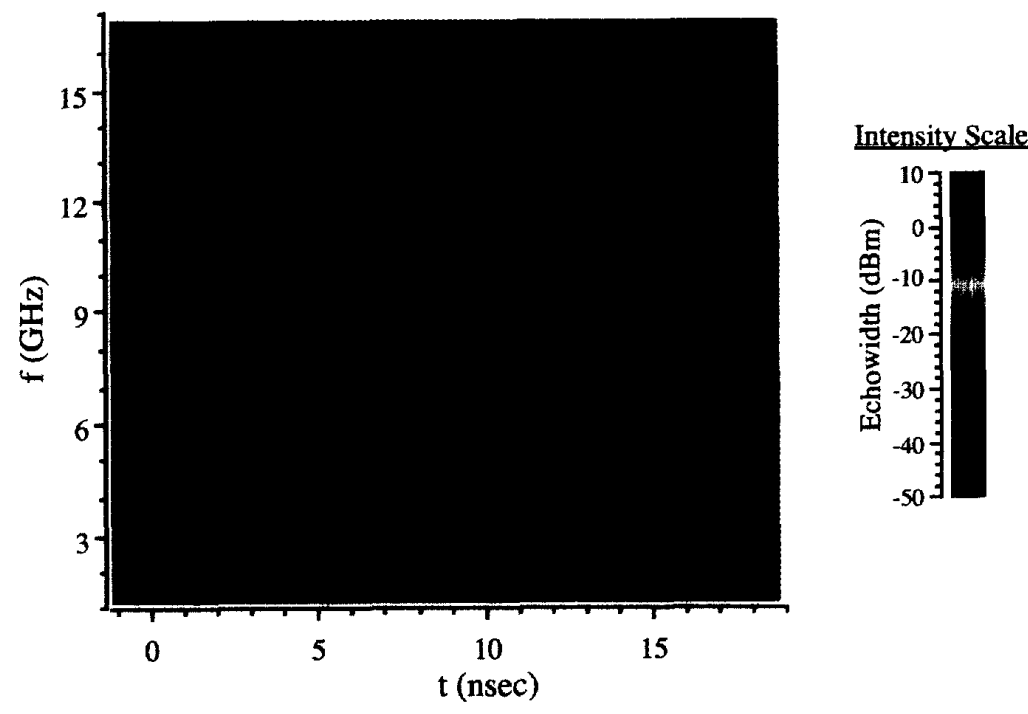

Fig. 3. Time-frequency image of the backscattered data (obtained via STFT).

arise. In addition, six cavity resonances are expected to be excited in the frequency range $0.5-18.0 \mathrm{GHz}$. Fig. 2(a) shows the frequencydomain scattered far-field generated by a two-dimensional moment method code based on an electric field integral equation formulation. A frequency step of $0.05 \mathrm{GHz}$ and a discretization width of 0.075 $\lambda_{0}$ were used.

Though the cavity resonances are expected to appear as sharp spikes in the frequency domain, they are overshadowed by the frequency behavior of the three scattering centers in the strip. Consequently, it is difficult to distinguish the resonances in Fig. 2(a). The time-domain data, shown in Fig. 2(b), are obtained by inverseFourier transforming the $0.5-18.0 \mathrm{GHz}$ data. A Kaiser window was applied to the frequency data before the transform. The locations of the three scattering centers (at $t \sim 0,2$, and $5 \mathrm{nsec}$ ) can be seen in Fig. 2(b), but the time-behavior of the cavity resonances makes it difficult to resolve each scattering center clearly. This is particularly true for the third scattering center which is excited before the resonances have significantly decayed.

Fig. 3 shows a time-frequency image of the scattered data obtained via the short-time Fourier transform (STFT). The three vertical lines correspond to the scattering centers, and the five visible horizontal lines correspond to the cavity resonances. It is possible to extract qualitative information such as the approximate frequency behaviors of the individual scattering centers and the resonance $Q$ 's from the STFT image. Due to the large number of features contained in the backscattered data, however, the image is blurry, making it difficult to resolve fine details.

\section{Parameter estimation in the TIME-Frequency Plane}

In our time-frequency super-resolution procedure, Prony's extraction is first applied in the frequency domain to locate scattering centers. It is clear that applying Prony's method to the entire frequency data of Fig. 2(a) will yield a poor fit since the raw data contains resonances. To circumvent this problem, we repeatedly apply Prony's method to many small windows of the raw data as shown in Fig. 4. Prony`s method will be successful for most of the window locations and will fail only when a window coincides with a resonant peak.

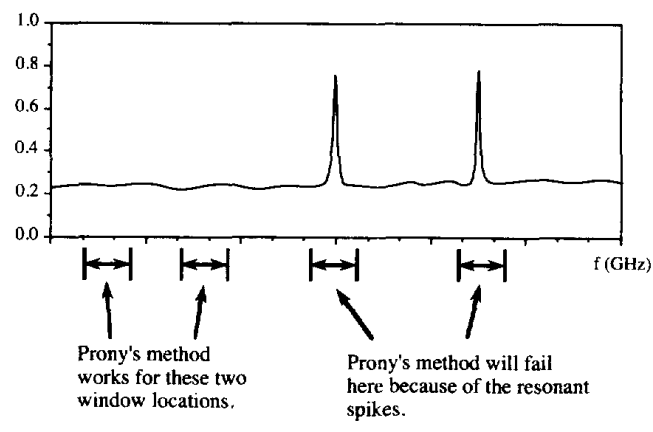

Fig. 4. Example of Prony's method being applied to small windows of backscattered data.

Prony's method will fit the raw field data $E_{k}$ within each window centered at $\alpha^{\prime} k$ and containing $L$ samples to the following model

$$
\hat{E}_{k}(\omega)=\sum_{m=1}^{M_{k}} A_{m, k} e^{-j \omega t_{m, k}}
$$

where $\hat{E}_{k}$ is the estimated field value, the $t$ 's are the estimated locations of the scattering centers, the A's are the estimated complex strengths of the scattering centers, and.$I_{k}$ is the number of scattering centers that Prony's method finds for the window centered at $\omega_{k}$. For our data, we use a $1 \mathrm{GHz}$ window containing $L=20$ samples.

To determine the true scattering center locations after applying Prony's method to all of the data segments, we define a weighting function to measure the goodness of the fit within each window as follows

$$
W_{k}=\exp \left\{-\gamma\left\|E_{k}-\hat{E}_{k}\right\| /\left\|E_{k}\right\|\right\} .
$$

In the above expression, $\left\|E_{k}-\hat{E}_{k}\right\| /\left\|E_{k}\right\|$ is the normalized error norm of the fit for the window centered at $\omega_{k}$ and $\gamma$ is chosen to be 25 . The true scattering centers are found by grouping with respect to time the $t_{m, k}$ from all the segments and carrying out a weighted average of their locations according to the above weight. It is clear that for windows in which Prony's method does poorly, the corresponding 


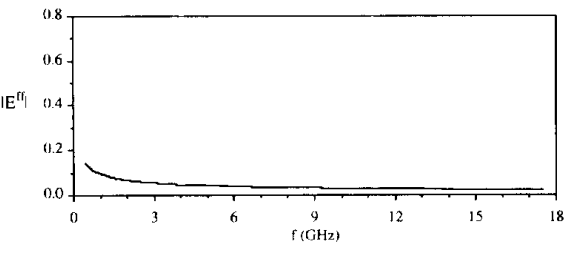

(a)

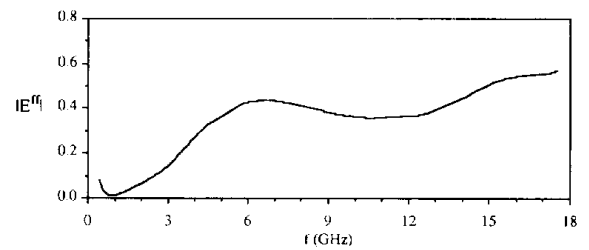

(b)

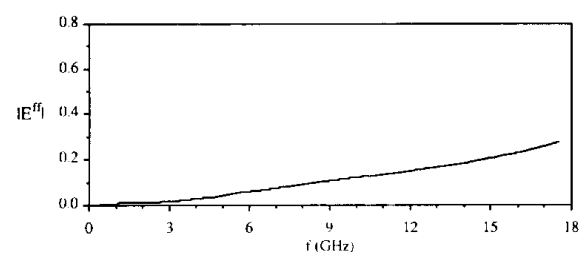

(c)

Fig. 5. Extracted amplitude behavior for the three scattering centers: (a) left edge, (b) cavity exterior, and (c) fin at right edge.

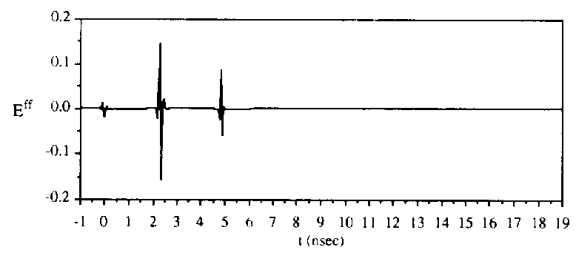

Fig. 6. Time domain plot of the fitted scattering center data.

weights $W_{k}$ will be small and prevent their associated values from contributing much to the result.

Depending on whether the scattering center is an edge, corner, or other more complex shape, its strength will be frequency dependent. A weighted least squares fit with respect to frequency of the values of $A_{m, k}$ for each scattering center is used to construct a smooth functional form of $A_{m}(\omega)$. A ninth-order polynomial function is used to approximate each $A_{m}$ in the entire frequency range. An important benefit of this global frequency description is that it allows us to go back and interpolate the scattering center strengths at those frequencies where Prony's method originally failed.

Shown in Fig. 5(a)-(c) are the frequency behaviors of the three scattering centers extracted by the sliding window Prony's fit. As predicted by diffraction theory, the first scattering center due to the left edge has a frequency dependence proportional to $k^{-1 / 2}$. Fig. 6 shows the behavior of the three scattering centers in the time domain. All three scattering centers are seen to be well localized in time. The third scattering center, in particular, has been completely separated from the resonant ringing which was present there in the original raw time data. Fig. 7 shows the remaining data after the scattering centers have been removed from the raw frequency data. Provided that the scattering centers have been properly located and accounted for, the remaining data will consist solely of a series of resonant peaks.

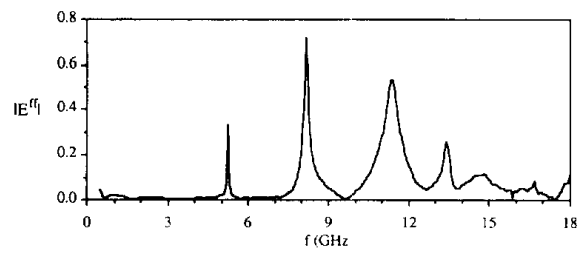

Fig. 7. Remaining resonant peaks after fitted scattering center data is subtracted from the raw frequency data.

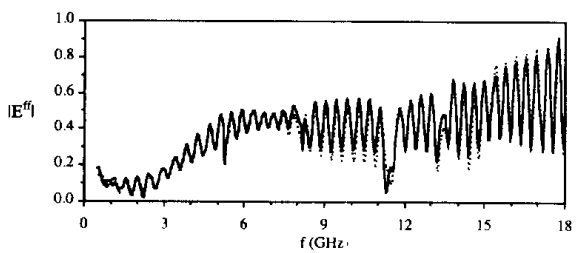

Fig. 8. Agreement between the original raw data and the combined, superresolved scattering center/resonance data.

To extract the natural resonance information, the remaining data are inverse Fourier transformed to the time domain. The sliding window Prony's procedure is then applied in the time domain to fit the complex-valued data to a model which is the dual of (1)

$$
\hat{e}_{1}(t)=\sum_{n=1}^{N_{i}} B_{n, i} t^{+j \omega_{n, i} t}
$$

in which the $\omega$ 's are the unknown resonance frequencies, the $B$ 's are their corresponding strengths, and $N_{i}$ is the number of resonances found for the window centered at $t_{i}$. By employing a similar weighted average scheme as in the scattering center extraction the true resonances are located. Then, by tracking the behaviors of $B_{n, i}$ with respect to time, other parameters associated with the resonance, such as attenuation factor $\left(a_{n}\right)$ and turn-on time $\left(\tau_{n}\right)$ are extracted

$$
\hat{e}(t)=\sum_{n=1}^{N} b_{n} e^{+j \omega_{n}\left(t-\tau_{n}\right)} e^{-a_{n}\left(t-\tau_{n}\right)} u\left(t-T_{n}\right)
$$

where $N$ is the number of true resonances, $b_{n}$ are the estimated strengths of the true resonances at turn-on, and $u\left(t-\tau_{n}\right)$ is the unit step function.

When the estimates for the five resonances and three scattering center behaviors are combined, the result is in good agreement with the original raw data as shown in Fig. 8. Fig. 9 shows a timefrequency plot of the parameterized backscattered data. Even though we have completely parameterized the data via a super-resolution technique, it is still useful to view the data in the time-frequency plane to provide insight not available by looking solely at the numerical estimates for each parameter. In displaying the super-resolved timefrequency imagery, we have chosen for each mechanism to appear as either a horizontal or vertical line exactly one pixel in width, although our image can be of nearly infinite sharpness. The intensities of the three vertical lines show that the three scattering centers are of differing strengths and have different frequency behaviors. The high- $Q$ resonances can be seen to be of much longer duration than the low $Q$ resonances.

\section{CONCLUSION}

In this paper, a time-frequency super-resolution procedure was presented. Prony's method was used in both the time and frequency domains to analyze the backscattered signal from a strip containing 


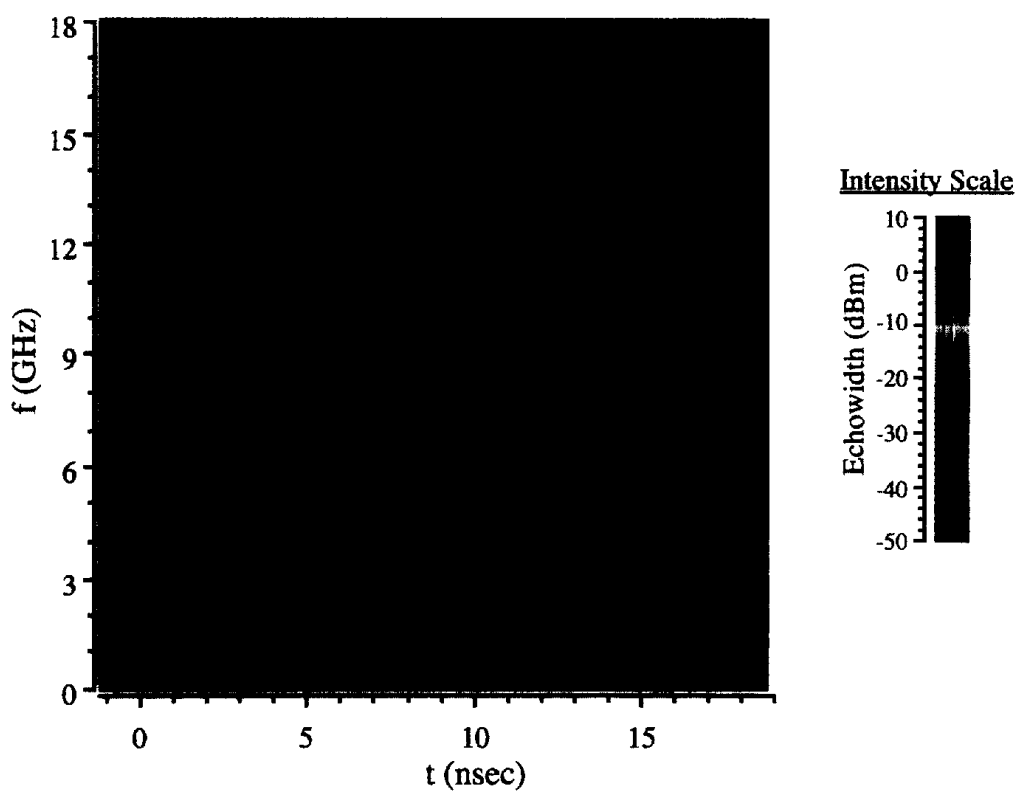

Fig. 9. Super-resolved time-frequency representation of the parameter fitted data.

a resonant cavity. Scattering center mechanisms were extracted by applying Prony's method to windowed sets of the frequency data. Cavity resonances were then found by applying Prony's method to windowed portions of the time data. Accurate models for each mechanism were obtained by weighing and averaging the results from successive applications of Prony's method. The resulting timefrequency display from the parameterized data is not constrained in resolution by the Fourier limit as are other time-frequency techniques.

Two important issues not addressed in this paper are noise and dispersion. First, Prony's method is known to be highly susceptible to noise. To combat the noise issue, a straightforward remedy is to replace the Prony engine during the processing with a more robust algorithm such as ESPRIT or MUSIC without altering the basic idea presented here. Such work has been carried out by us and will be reported in [9]. Second, to achieve a more complete parameterization in the time-frequency plane for general targets, dispersive mechanisms such as surface waves and structural modes will need to be addressed. Extension of our technique to data containing dispersive mechanisms is currently under investigation [10], [11].

\section{ACKNOWLEDGMENT}

The authors thank Dr. M. P. Hurst of McDonnell Douglas for providing the Prony's routine used in this work and Prof. G. Xu for helpful discussions.

\section{REFERENCES}

[1] H. Ling and H. Kim, "Wavelet analysis of backscattering data from an open-ended waveguide cavity," IEEE Microwave Guided Wave Lett., vol. GWL-2, pp. 140-142, Apr. 1992.

[2] A. Moghaddar and E. K. Walton, "Time-frequency-distribution analysis of scattering from waveguide cavities," IEEE Trans. Antennas Propagat., vol. 41, pp. 677-680, May 1993.

[3] J. Moore and H. Ling, "Time-frequency analysis of the scattering phenomenology in finite dielectric gratings," Microwave Opt. Tech. Lett., vol. 6, pp. 597-600. Aug. 1993.
[4] H. Ling, J. Moore, D. Bouche, and V. Saavedra, "Time-frequency analysis of backscattered data from a coated strip with a gap," IEEE Trans. Antennas Propagat., vol. 41, pp. 1147-1150, Aug. 1993.

[5] A. J. Poggio, M. L. Van Blaricum, E. K. Miller, and R. Mittra, "Evaluation of a processing technique for transient data," IEEE Trans. Antennas Propagat., vol. AP-26, pp. 165-173, Jan. 1978.

[6] R. Roy, A. Paulraj, and T. Kailath, "ESPRIT-A subspace rotation approach to estimation of parameters of cisoids in noise," IEEE Trans. Acoust., Speech, Signal Processing, vol. ASSP-34, pp. 1340-1342, Oct. 1986.

[7] M. L. Van Blaricum and R. Mittra, "A technique for extracting the poles and residues of a system directly from its transient response." IEEE Trans. Antennas Propagat, vol. AP-23, pp. 777-781, Nov. 1975.

[8] M. P. Hurst and R. Mittra, "Scattering center analysis via Prony's method," IEEE Trans. Antennas Propagat., vol. AP-35, pp. 986-988, Aug. 1987.

[9] J. Moore, L. Trintinalia, H. Ling, and G. Xu, "Super-resolved timefrequency processing of wideband radar echo using ESPRIT," Microwave Opt. Tech. Lett., vol. 9, pp. 17-19, May 1995.

[10] J. Moore and H. Ling, "Super-resolved time-frequency processing of surface wave mechanisms contained in wideband radar echo," to appear in Microwave Optical Tech. Lett., Aug. 1995.

[11] L. C. Trintinalia and H. Ling, "Super-resolved time-frequency parameterization of electromagnetic scattering mechanisms due to structural dispersion," submitted to Microwave Optical Tech. Lett., Apr. 1995. 